\title{
Deliberate versus intrinsic disorder in photonic crystal nanocavities investigated by resonant light scattering
}

\author{
S. L. Portalupi, ${ }^{1}$ M. Galli, ${ }^{1}$ M. Belotti, ${ }^{1}$ L. C. Andreani, ${ }^{1}$ T. F. Krauss, ${ }^{2}$ and L. O' Faolain ${ }^{2}$ \\ ${ }^{1}$ Dipartimento di Fisica 'A.Volta,' Università degli Studi di Pavia, Via Bassi 6, IT-27100 Pavia, Italy \\ ${ }^{2}$ Scottish Universities Physics Alliance (SUPA), School of Physics and Astronomy, University of St. Andrews, \\ Fife KY16 9SS, United Kingdom \\ (Received 14 March 2011; published 12 July 2011)
}

\begin{abstract}
We report a study of photonic crystal nanocavities as a function of lattice disorder, which is implemented by deliberate radius variations of the holes forming the photonic crystal. Using cross-polarized laser light scattering, we demonstrate that lattice disorder affects the cavity mode symmetry, as is crucially evidenced by measuring scattering resonances for different sample orientations and explained by group-theoretical analysis together with calculations of the mode profile. We also quantify how the increase of lattice disorder leads to a reduction of the cavity $Q$ factors and to a spread of both $Q$ factors and resonance wavelengths. The trends as a function of disorder and for different radii distributions are well reproduced by theoretical calculations when both intentional (deliberate) and intrinsic (residual) disorder are taken into account. The results shed light on the effects of disorder on cavity resonances and on the interplay between intentional and intrinsic disorder, yielding reliable estimations of residual disorder (which ultimately affects technology limits) from optical measurements. Interestingly, the disorder values derived from the variation of the $Q$ factor are lower than those derived from the wavelength spread: this suggests the occurrence of subtle interhole correlation effects that turn out to be beneficial for cavity $Q$ factors.
\end{abstract}

DOI: 10.1103/PhysRevB.84.045423

PACS number(s): 42.70.Qs, 78.20.Bh, 78.67.Pt

\section{INTRODUCTION}

In recent years, high- $Q$ optical nanocavities have been widely used in many different scientific areas such as nanophotonics, ${ }^{1-6}$ cavity quantum electrodynamics, ${ }^{7,8}$ nonlinear enhancement, ${ }^{9}$ and biochemical sensing. ${ }^{10,11}$ The interest is motivated by the possibility to concentrate the electromagnetic field in a very small spatial region, increasing the radiationmatter interaction. In this way, physical phenomena on the nanoscale level can be studied. It has been demonstrated that by using planar photonic crystal (PhC) nanocavities, obtained by two-dimensional patterning of a slab waveguide, ultrahigh- $Q$ factors $^{2-5}$ can be realized. The key to obtaining high $Q$ 's is related to the optimization of the position and radius of some holes constituting the photonic crystal. ${ }^{12-14}$ For this reason, the positions and the radii of holes forming the cavity, and the photonic crystal in general, become very important parameters. But, during fabrication, some unintentional lattice disorder is invariably introduced, i.e., an unexpected variation in the hole radii forming the $\mathrm{PhC}$. This motivates a systematic study of the effects of lattice disorder on the cavity mode in order to understand how it modifies the cavity properties such as the $Q$ factor and the mode symmetry.

The quality factors of passive (nonemitting) $\mathrm{PhC}$ slab cavities can be determined by measuring transmission through a waveguide optically coupled to the nanocavity, by measuring light released from the cavity in the channel-drop filter geometry, or else by resonant scattering of light. The latter technique, first introduced in Ref. 15 for two-dimensional $\mathrm{PhC}$ slabs and later applied to nanocavities, ${ }^{16,17}$ consists of measuring the reflection of light from the surface of the $\mathrm{PhC}$ slab with crossed polarizations for the incident and outgoing beams: the optical signal has a strong resonance enhancement at the wavelength of a cavity mode. This technique is highly valuable as a tool for systematic studies of cavity $Q$ factors while varying the $\mathrm{PhC}$ lattice geometry (e.g., as a function of hole positions and radii) without the need of using coupling waveguides. Also, cross-polarized light scattering with the resulting Fano lineshapes ${ }^{18}$ depends strongly on coupling of the cavity mode with the incoming and outgoing fields, which in turn depends on the cavity mode symmetry and may be affected by disorder. Thus, a study of the effects of disorder on cavity modes may also be useful in order to shed light on physical mechanisms determining the light-scattering phenomenon.

In this paper, we consider $L 3$ cavities in silicon $\mathrm{PhC}$ slabs, obtained by removing three holes along the $\Gamma-K$ direction of the triangular lattice, and perform a systematic study of the effects of disorder on cavity modes by means of resonant light scattering. To this purpose, we introduce a deliberate amount of disorder by modifying the hole radii according to a Gaussian distribution with r.m.s. deviation $\sigma$, and investigate the resulting properties as a function of $\sigma$ in a lithographically controlled way. The paper is organized as follows: In Sec. II, we briefly describe sample fabrication, the optical technique, and the theoretical methods, including a group-theoretical analysis of coupling with external photons based on cavity mode symmetry. In Sec. III, we analyze the effect of disorder on resonant light scattering. In Sec. IV, we present systematic results for cavity $Q$ factors with different hole geometries as a function of increasing disorder. Section $V$ contains concluding remarks. The results will be shown to be useful in order to quantify the interplay between intentional (deliberate) and intrinsic (technology-limited) disorder, with a precise estimation for the residual effect of intrinsic disorder in the investigated $\mathrm{PhC}$ cavities. 

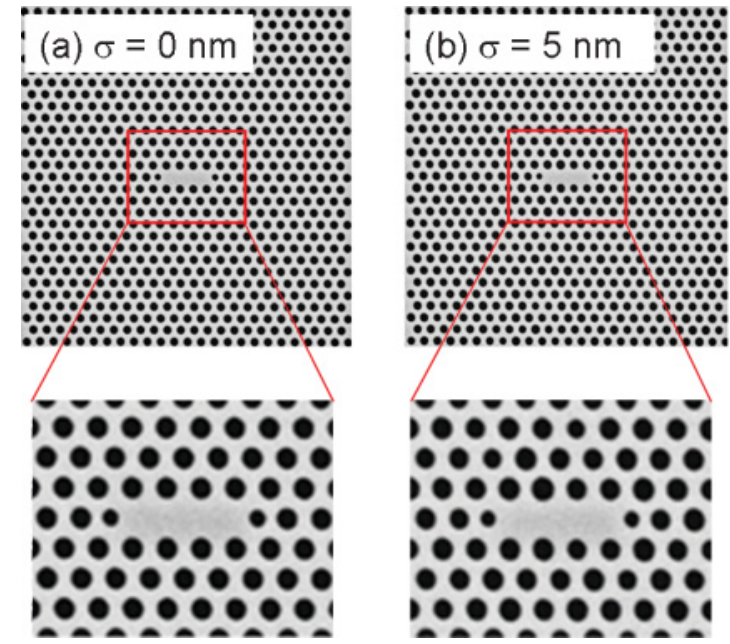

\section{SAMPLES AND METHODS}

\section{A. Sample fabrication}

The nanocavities were fabricated from a Silicon-onInsulator wafer (SOITEC, $220 \mathrm{~nm}$ nominally thick silicon on $2000 \mathrm{~nm}$ of silica) using electron beam lithography (hybrid ZEISS GEMINI 1530/RAITH ELPHY system) and reactive ion etching. Then, the silica was removed by means of wet etching via a vapor hydrofluoric-acid method in order to obtain airbridged $\mathrm{PhC}$ nanocavities. The procedure, similar to that of Refs. 19 and 20, is optimized for high-quality photonic crystals. A $50-\mu \mathrm{m}$ size writing field was used and each cavity had the same location in the write field, avoiding any possible variations caused by the write field distribution. All the fabricated cavities have lattice constant $a=420 \mathrm{~nm}$ and hole radii of approx $126 \mathrm{~nm}(r / a=0.3)$. Optimization of radius and position of the holes adjacent to the cavity ${ }^{12-14}$ (hole shrinking $\Delta r / a=-0.06$ and shifting $\Delta x / a=0.14$ to 0.2 ) has been performed in order to obtain high- $Q$ factors.

In order to study the effects of lattice disorder, cavities with different hole radii distribution, that is, with different degree of disorder, were fabricated. The amount of lattice disorder is quantified by the parameter $\sigma$, defined as the r.m.s deviation of the Gaussian distribution of the hole radii. Figure 1 shows scanning electron microscope (SEM) images of three cavities with different values of $\sigma$. The large cavity area $(10 \mu \mathrm{m} \times$ $10 \mu \mathrm{m})$ is realized in order to reduce in-plane losses to a negligible level. The first image refers to a cavity with zero intentional disorder (a small unintentional contribution to disorder is still present, as discussed later). The last one refers to a cavity with the highest disorder parameter $\sigma=10 \mathrm{~nm}$; it is noteworthy that the variation in the hole radii is clearly visible in the SEM image. Cavities with disorder parameter $\sigma$ ranging from 0 to $10 \mathrm{~nm}$ ( $1 \mathrm{~nm}$ step) were fabricated and optically characterized.

\section{B. Experimental technique}

The optical characterization was carried out using crosspolarized laser light scattering. ${ }^{15,16}$ The scheme of the experimental setup is shown in Fig. 2(a). Light from a continuous-wave $(\mathrm{CW})$ tunable laser is linearly polarized by the polarizer $P$ and focused on the sample by means of a polarization-maintaining high numerical aperture objective (NA =0.8). The light reflected by the sample is collected using a beam splitter and analyzed by the analyzer $A$ in cross polarization with respect to the polarizer $P$. Figure 2(b) shows the orientation of the cavity with respect to polarizer $P$ : the cavity axis (dotted line) forms an angle $\phi$ with the polarizer and this angle can be controlled in the experiments. In the figure, this angle equals $45^{\circ}$, which is the configuration that maximizes cross-polarized light scattering, as will be shown in Sec. IV. In order to increase the signal-to-noise ratio, incident light is modulated using an optical chopper with the modulation frequency taken as reference for a lock-in amplifier. Once the incident light polarization is chosen, the analyzer is oriented in order to minimize the signal on detector when the laser light frequency is off resonance with respect to the cavity mode. This was shown to yield the maximum on-off ratio of the resonant signal, with the same $Q$ factor being obtained from a Fano-lineshape analysis. ${ }^{16}$

Figure 3 shows typical scattering spectra (black dots) and best fits with Fano curves ${ }^{16,18}$ (red lines) for cavities with different degree of disorder $(\sigma=0,5,9 \mathrm{~nm}$, respectively). These spectra were taken for a rotation angle $\phi=45^{\circ}$, which maximizes the signal. As it can be seen, the first effect of intentional lattice disorder is a reduction of the quality factor. The first cavity [Fig. 3(a)], in which the intentional disorder

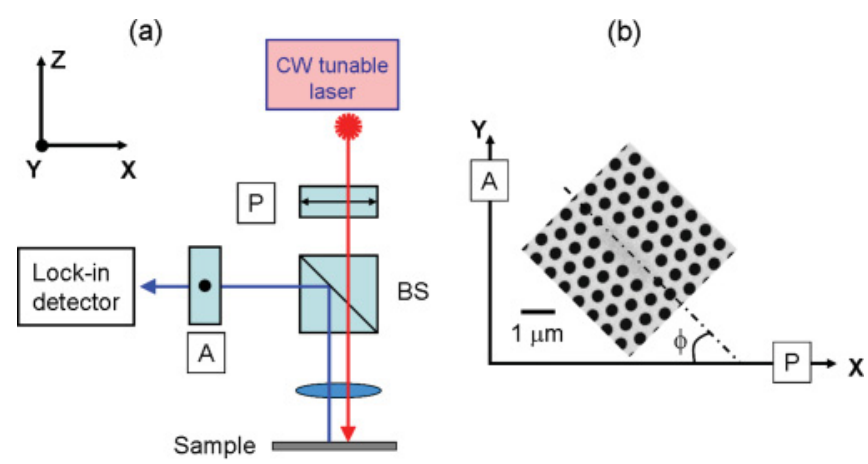

FIG. 2. (Color online) (a) Scheme of the experimental setup for cross-polarized CW light scattering measurements. (b) SEM image of an $L 3 \mathrm{PhC}$ nanocavity, showing its orientation with respect to polarizer and analyzer. 

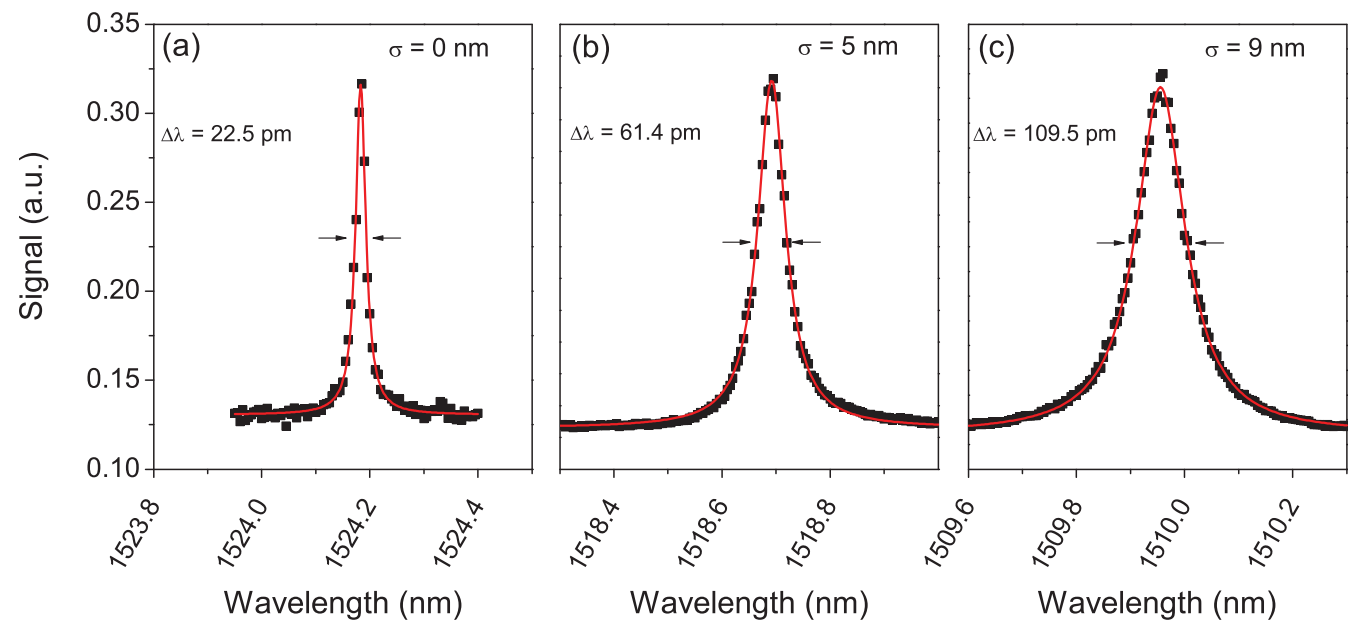

FIG. 3. (Color online) Cross-polarized scattering spectra (square dots) for three cavities with $a=420 \mathrm{~nm}, r / a=0.3, \Delta x / a=0.16$, $\Delta r / r=-0.06$ but with different amount of disorder ( 0,5 , and $9 \mathrm{~nm}$, respectively). Best fits with Fano lineshapes are also shown (continuous lines).

is zero, has a quality factor of 67700 , meaning a full width at half-maximum (FWHM) of $\Delta \lambda=22.5 \mathrm{pm}$. Increasing the intentional disorder $[\sigma=5 \mathrm{~nm}$, Fig. 3(b)] reduces the $Q$ to 24800 with the FWHM equal to $\Delta \lambda=61.4 \mathrm{pm}$. A further increase of disorder [Fig. 3(b), $\sigma=9 \mathrm{~nm}$ ] leads to a further decrease of the quality factor (13900) and increase of FWHM $(\Delta \lambda=109.5 \mathrm{pm})$. A detailed study of the effect of disorder on $Q$ will be presented in Sec. III.

\section{Theory}

Numerical results for cavity mode profiles and $Q$ factors are calculated with a guided-mode expansion (GME) method that consists of expanding the electromagnetic field on the basis of guided modes of an effective homogeneous waveguide and calculating coupling to radiative modes by perturbation theory. ${ }^{21}$ To treat an L3 cavity, a supercell is introduced along the cavity axis $\Gamma K$ and along the perpendicular direction $\Gamma M$. Typical supercell dimensions are $10 a$ along $\Gamma K$ and $4 \sqrt{3} a$ along $\Gamma M$. The quality factor is obtained as $Q=\omega /[2 \operatorname{Im}(\omega)]$, where $\omega$ is the cavity mode frequency and $\operatorname{Im}(\omega)$ is its imaginary part. Results for $Q$ factors of $L 3$ cavities with optimized local geometry (i.e., shift and shrink of nearby holes in the absence of disorder) are given in Ref. 13 and are close to the results obtained with a three-dimensional (3D) frequency-domain modal method. ${ }^{14}$ The effect of radial disorder on cavity modes is treated within the GME method by assuming a random distribution of the hole radii $r$ according to $P(r) \propto \exp \left[-(r-\bar{r})^{2} /\left(2 \sigma^{2}\right)\right]$ within the same supercell. Notice that, in this paper, the zero-order calculation (i.e., before applying perturbation theory for coupling to radiative modes) is made with the hole's radii in the chosen supercell being randomly distributed with variance $\sigma$, thus, the effect of disorder is treated in a nonperturbative way. It is only the very weak coupling to radiation modes of the $\mathrm{PhC}$ slab that is treated perturbatively: this approximation, which is validated in Ref. 21 , is very well justified for high- $Q$ modes that have a small coupling to radiative $\mathrm{PhC}$ slab modes. The present approach is suitable and necessary when the disorder parameter $\sigma$ becomes larger than a few nm; indeed, the inverse $Q$ factor as a function of $\sigma$ deviates from the perturbative $\propto \sigma^{2}$ behavior found in previous work..$^{22,23}$ Also, the cavity mode profiles and local electromagnetic field variation in the disordered lattice are fully calculated, as shown below.

We start the discussion of resonant light scattering by a group-theoretical analysis of the symmetry of cavity modes, along the lines of Ref. 24. This allows us to discuss coupling of cavity modes to the radiation field, concerning polarization properties and the mechanism of resonant scattering, in the most general way. The point group of an L3 cavity in the triangular lattice is $C_{2 v}$ [considering only two-dimensional (2D) operations] or $D_{2 h}$ [considering full 3D symmetry operations]. In order to determine the symmetry of the ground cavity mode, in Fig. 4 we show the field components $E_{x}$ and $E_{y}$ with respect to the cavity axes (notice that the axes $x$ and $y$ in Fig. 4 are different from the polarizer and analyzer axes $X, Y$ in Fig. 2). It can be seen that the component $E_{y}$ is larger and more concentrated in the cavity region; indeed, this is the dominant component of the index-guided mode in the corresponding $W 1$ waveguide, which is confined in the region of the three missing holes to yield the ground $L 3$ cavity mode.

Now, the behavior of a field component $E_{\xi}(\mathbf{r})$ under the action of a symmetry operation $\hat{O}$ follows from the transformation properties of the vector component $E_{\xi}$ and of its spatial coordinate $\mathbf{r}$, i.e.,

$$
\hat{O} E_{\xi}(\mathbf{r})=\left(\hat{O} E_{\xi}\right)\left(\hat{O}^{-1} \mathbf{r}\right)
$$

Considering, for example, mirror reflection with respect to the $x z$ plane, represented by the operator $\sigma_{x z}$, the $E_{y}$ vector component is odd under the action of $\sigma_{x z}$, while its spatial dependence is even; thus,

$$
\sigma_{x z} E_{y}(\mathbf{r})=\left(\sigma_{x z} E_{y}\right)\left(\sigma_{x z}^{-1} \mathbf{r}\right)=\left(-E_{y}\right)(x,-y, z)=-E_{y}(\mathbf{r})
$$

and the component has a -1 eigenvalue under mirror-reflection operator $\sigma_{x z}$. The other vector component $E_{x}$ is even under the 

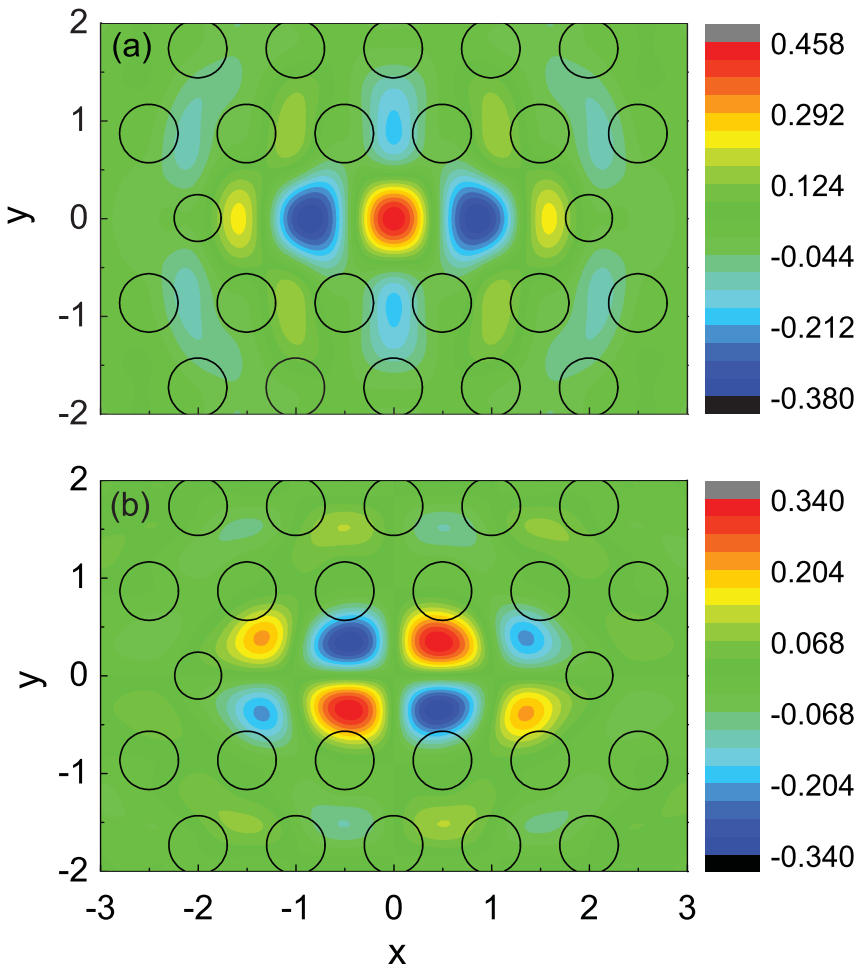

FIG. 4. (Color online) Imaginary part of the electric field (a) $E_{y}$ and (b) $E_{x}$.

mirror-reflection operator $\sigma_{x z}$, while its spatial dependence is odd, also yielding a -1 global eigenvalue. This allows us to conclude that the cavity mode is globally odd under the action of $\sigma_{x z}$. By proceeding in the same way for all the symmetry operations of the $3 \mathrm{D}$ point group $D_{2 h}$ (which of course includes all operations of $C_{2 v}$ as a subset), we get the transformation shown in Table I.

By comparing with the character table of Refs. 24 and 25, we recognize that the ground $L 3$ cavity mode behaves as the $B_{2 u}$ irreducible representation (for $D_{2 h}$ ) or as $B_{2}$ (for $C_{2 v}$ ). These are also the irreducible representations of the $y$ coordinate or the $d_{y}$ dipole component; thus, the ground $L 3$ cavity mode has $y$ symmetry and, in the $2 \mathrm{D}$ photonic crystal without disorder, can only be excited by a $y$-polarized field component. Since cross-polarized scattering requires coupling of the cavity mode with incoming and outgoing fields at crossed polarizations, this implies that the cavity mode can not appear in resonant scattering when the cavity axes are at an angle of $0^{\circ}$ or $90^{\circ}$ with respect to the polarizer and analyzer axes. Indeed, for a rotation angle $\phi=0^{\circ}$ [see Fig. 2(b) for the definition of the angle $\phi$ ], the $y$ axis is aligned with the analyzer and the cavity mode is coupled to the outgoing field,

TABLE I. Character table for the ground mode of an $L 3$ cavity in the absence of disorder. The operators $E, C, i$, and $\sigma$ stand for identity, $\pi$ rotation, inversion, and mirror reflection.

\begin{tabular}{cccccccccc}
\hline \hline$D_{2 h}$ & 110 \\
\hline & $E$ & $C_{2}(z)$ & $C_{2}(y)$ & $C_{2}(x)$ & $i$ & $\sigma_{x y}$ & $\sigma_{x z}$ & $\sigma_{y z}$ & \\
\hline 1 & -1 & 1 & -1 & -1 & 1 & -1 & 1 & $B_{2 u}(y)$ \\
\hline \hline
\end{tabular}
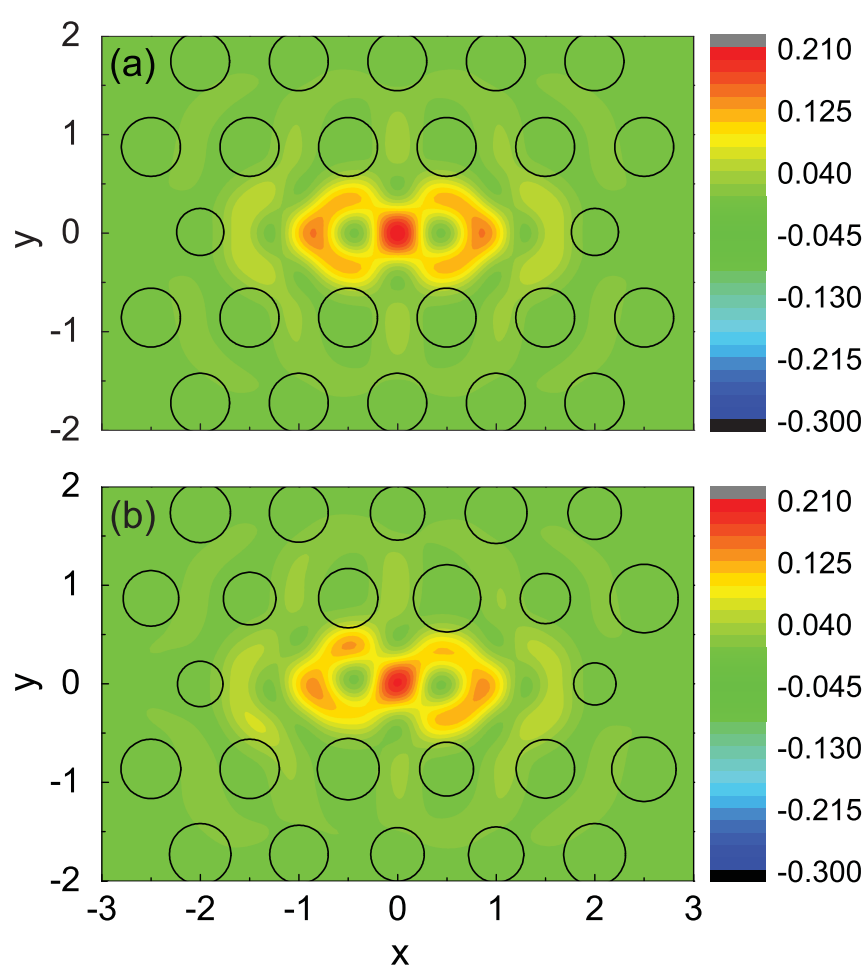

FIG. 5. (Color online) Squared electric field profiles for the cases of (a) nominal zero disorder and (b) nominal $\sigma=10 \mathrm{~nm}$ disorder. The random variations of the radii can be noticed in (b).

but not to the incoming field; on the contrary, for $\phi=90^{\circ}$, the $y$ axis is aligned with the polarizer and the cavity mode is coupled to the incoming field, but not to the outgoing one. The $\phi=45^{\circ}$ configuration is the one that maximizes the resonant scattering signal, as it is the best compromise for simultaneous coupling of the incoming and outgoing fields.

These symmetry properties are changed in the presence of lattice disorder. Indeed, a variation in the hole radii forming the lattice leads to a breaking of the $D_{2 h}$ symmetry. Figure 5 shows the squared field profiles for two different cases: on top the field is calculated for $\sigma=0 \mathrm{~nm}$ (no disorder) and on bottom for $\sigma=10 \mathrm{~nm}$ (highest disorder). It is clear that the presence of lattice disorder breaks the symmetry of the lattice and of the mode profile. In the presence of disorder, there is no symmetry operation beyond the identity and the selection rules previously derived for the case of zero disorder are broken. Thus, we expect that, on increasing the degree of disorder, the cavity mode can couple to incoming or outgoing light for any polarization, and that resonant light scattering becomes observable for all rotation angles $\phi$. The experimental consequences of these results are studied in the next section.

\section{EFFECT OF INTENTIONAL DISORDER ON LIGHT SCATTERING}

In order to study the effects of intentional disorder on light scattering, measurements on samples with different orientations were made. In detail, Fig. 6 shows the three different orientations of the sample chosen in our measurements. As 

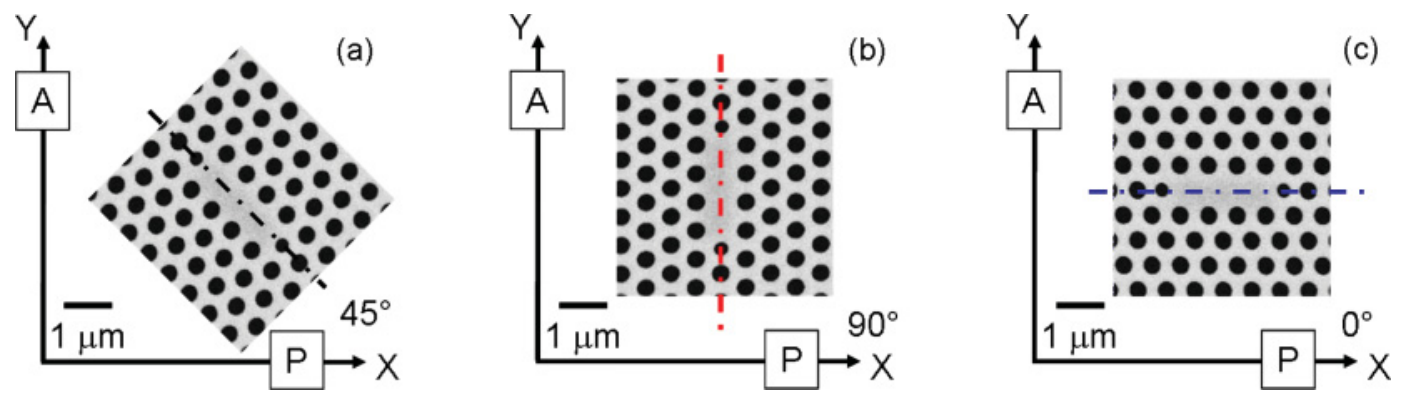

FIG. 6. (Color online) The figure shows three different orientations of the cavity axis with respect to the polarizer $P$ : (a) $45^{\circ}$, (b) $90^{\circ}$, and (c) $0^{\circ}$.

explained in Sec. II C, the $45^{\circ}$ configuration represents the best compromise for simultaneous coupling with incoming and outgoing fields. For this reason, the signal should be stronger with respect to every other orientation, in particular, with respect to the $90^{\circ}$ or $0^{\circ}$ configurations, for which the coupling is only with incoming or outgoing field but not both simultaneously: in these cases $\left(90^{\circ}\right.$ and $\left.0^{\circ}\right)$, the signal should be a flat background. The situation is different in the presence of lattice disorder: As suggested by the mode profile calculations, the disorder leads to a different coupling with incoming and outgoing fields, leading to the expectation of a scattering signal also for $90^{\circ}$ and $0^{\circ}$.

In order to demonstrate this, measurements in the three configurations were made. Figure 7 shows scattering spectra for increasing degrees of disorder $(\sigma=0,5,10 \mathrm{~nm})$ and for the three rotation angles $\phi=45^{\circ}, 90^{\circ}$, and $0^{\circ}$. In Fig. 7(a), nominal $\sigma=0$, the signal for the $45^{\circ}$ orientation (square dots) is much stronger than for $90^{\circ}$ and $0^{\circ}$ (triangular and circular dots, respectively). However, we can notice that in the $90^{\circ}$ and $0^{\circ}$ cases, a scattering signal is present, even if very weak. There are two reasons for the presence of a signal: First, a small amount of unintentional lattice disorder is always present, ${ }^{16}$ even in the case of nominal $\sigma=0$; second, the cavity axis can not be perfectly oriented at $90^{\circ}$ or $0^{\circ}$ angles. The situation changes with the introduction of intentional lattice disorder [Figs. 7(b) and 7(c)]: The ratio between the spectra changes significantly and, for $\sigma=10 \mathrm{~nm}$, the spectra for the three angles are comparable to each other.
In the cases with appreciable disorder, $\sigma=5$ or $10 \mathrm{~nm}$, the presence of a clear signal for all three orientations means that there is simultaneous coupling of the ground $L 3$ cavity mode with incoming and outgoing fields. In other words, these results demonstrate that lattice disorder breaks the in-plane symmetry of the system, as expected from calculations of the fundamental mode previously shown in Fig. 5. At the same time, the results demonstrate that the cross-polarized lightscattering technique can be operated in two different regimes: In the case of weak disorder, the signal is very weak for $0^{\circ}$ and $90^{\circ}$ and is maximum for $\phi=45^{\circ}$, which is the configuration that maximizes coupling of the cavity mode with the incoming and outgoing fields. On the other hand, when disorder is strong, resonant scattering can be easily observed for any orientation as coupling between the cavity mode and ingoing or outgoing field is provided by the disorder through breaking of planar symmetry of the PhC cavity.

\section{EFFECT OF INTENTIONAL DISORDER ON CAVITY $Q$ FACTORS}

Aside from the variation of the mode symmetry, lattice disorder influences also the quality factor of the cavity mode, as was shown in Fig. 3. To study the effect on the $Q$ in a systematic way, we optically characterized six series of cavities with different nearby hole shifts $\Delta x / a$ (equal to 0.14 , $0.16,0.17,0.18,0.19$, and 0.2 , respectively) and with disorder parameter $\sigma$ ranging from 0 to $10 \mathrm{~nm}$. Referring to Fig. 8,
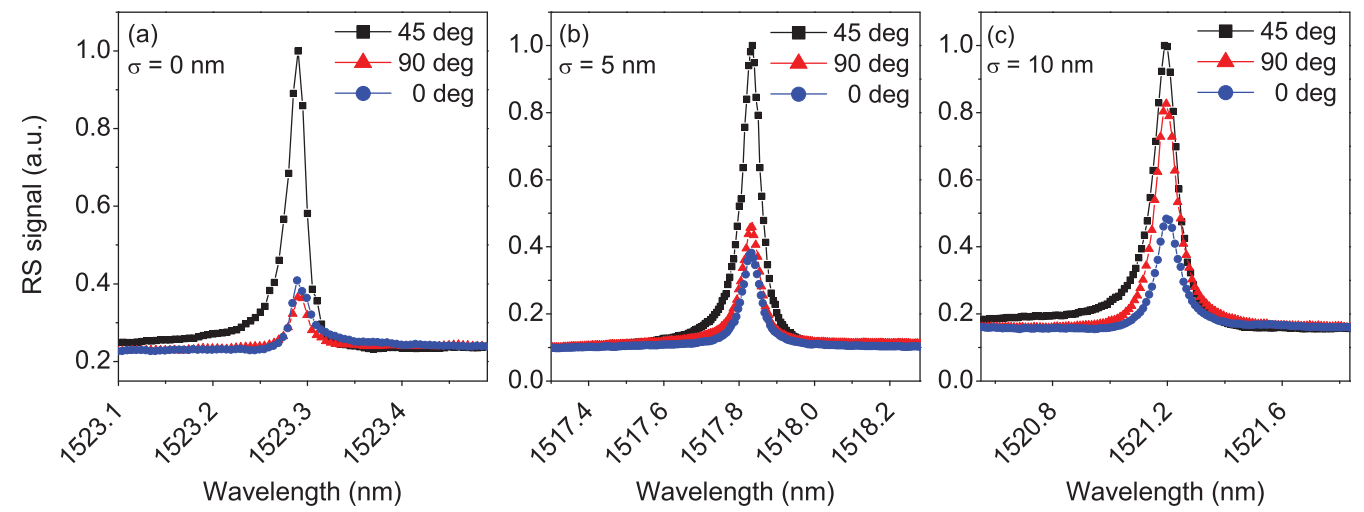

FIG. 7. (Color online) Scattering spectra for three cavities with $\Delta x / a=0.16$ and disorder parameter (a) $\sigma=0 \mathrm{~nm}$, (b) $\sigma=5 \mathrm{~nm}$, and (c) $\sigma=10 \mathrm{~nm}$, respectively. Spectra are measured for three different sample orientations: $45^{\circ}$ (square dots), $90^{\circ}$ (triangular dots), and $0^{\circ}$ (circular dots). 

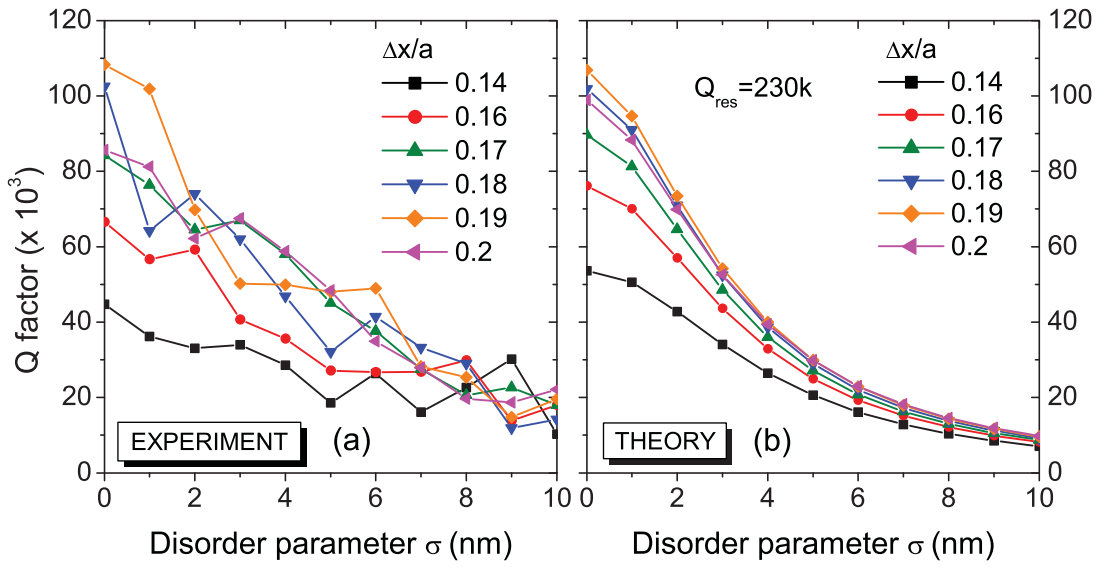

FIG. 8. (Color online) (a) Experimental behavior of the $Q$ factors versus the disorder parameter $\sigma$ for six series of cavities with different nearby hole shifts $\Delta x / a$. (b) Theoretical calculation of $Q$ versus $\sigma$, assuming an intrinsic $Q_{\text {res }}=230 \times 10^{3}$ (see text). It should be noted that each experimental datapoint corresponds to a different disorder distribution, whereas the distribution is the same for each of the theoretical curves and is scaled with $\sigma$. each series of cavities is characterized by a fixed hole shift and different amounts of disorder. All measurements shown below were made with a sample orientation of $45^{\circ}$.

In Fig. 8(a), one can notice that, as might be expected, there is a reduction in the $Q$ factor for every series of cavities with increasing disorder parameter $\sigma$. The relative reduction is stronger for series in which the $Q$ value for zero intentional disorder is higher, i.e., the cavities with lower $Q$ factor are more robust to lattice disorder. In Fig. 8(b), we show the theoretical points, obtained as follows: For a particular disorder distribution, the $Q$ factor is calculated for each $\sigma$ and $\Delta x / a$, giving a design value $Q_{\text {design. The effect }}$ of unintentional, residual disorder is taken into account by using the phenomenological formula ${ }^{22,26} 1 / Q=1 / Q_{\text {design }}+$ $1 / Q_{\text {res }}$, where the term $Q_{\text {res }}$ is obtained from the best fit of data and is found to be $Q_{\text {res }}=230 \times 10^{3}$. Figure 8 shows that the reduction of the quality factor for increasing $\sigma$ is in good agreement with the theoretical calculation. Notice that the trend as a function of nearby hole shift $\Delta x / a$ is also well reproduced. Following Ref. 22, we can estimate the residual disorder by using the result

$$
1 / Q_{\mathrm{res}}=A(\sigma / a)^{2},
$$

where $A$ is calculated ${ }^{27}$ to be 0.62 for the present cavities, and obtain $\sigma=1.1 \mathrm{~nm}$. This is a measure of the residual r.m.s. variation of hole radii in the present sample.

As it is possible that different disorder distributions give very different effects (even for the same $\sigma$ ), we fabricated and measured cavities with many disorder distributions in order to verify that the reduction of the $Q$ factor as a function of $\sigma$ is a general feature and not related to a particular realization of disorder. All the new samples have the same geometrical parameters $(a=420 \mathrm{~nm}, r / a=0.3, \Delta r / a=-0.06, \Delta x / a=$ $0.16)$ but different disorder distributions, i.e., different sets of random numbers were used to generate the radii. The values for $\sigma$ for this new set of samples are restricted to 0,5 , and $10 \mathrm{~nm}$. We can notice in Fig. 9(a) that, similar to before, there is an overall reduction of the quality factors by increasing the disorder, but we can also recognize a considerable spread of values for a fixed $\sigma$. In Fig. 9(b), we report the theoretical calculation of $Q$ 's versus disorder for 15 distributions (labeled by the seed for random number generator). Again, we use the formula $1 / Q=1 / Q_{\text {design }}+1 / Q_{\text {res }}$ and find $Q_{\text {res }}=700 \times$ $10^{3}$, which indicates the improved quality of the new sample.
By using Eq. (3), we obtain a residual disorder parameter $\sigma=0.64 \mathrm{~nm}$, considerably lower than in the previous sample because of improved fabrication.

The overall behavior and the spread of values is quite similar in experiment and theory; however, the theoretical $Q$ 's and the corresponding spreads tend to be smaller than the experimental values. This discrepancy is probably due to imperfections in the implementation of the disordered radii during electron-beam lithography. During the exposure, it is possible that the backscattering of electrons from the substrate $^{28}$ has a slightly homogenizing effect on the resulting hole radii. When a large hole and a small hole are adjacent to one another, stray electrons from the large hole enlarge the radius of the small hole by an amount that is proportional to the area of the larger hole (i.e., to the number of incident electrons). In return, the small hole enlarges the large hole by an amount that is proportional to its own area. Thus, this phenomenon may correlate the radii of neighboring holes, reducing the randomness and increasing the experimental $Q$ factors relative to the calculations with uncorrelated disorder. Due to the nanometer scale of this disorder, observing this correlation using analysis of SEM images is very challenging.

Looking at the values without intentional disorder $(\sigma=0)$, we would expect the $Q$ 's to be the same for all the cavities, as is indeed the case in the theoretical results; in the experiments, however, the reproducibility limits of the fabrication processes can lead to different $Q$ values for nominally identical cavities. Indeed, as we can see in Fig. 9(a), the cavities with no intentional disorder show a noticeable difference in the $Q$ factors. This spread in $Q$ values corresponds to the theoretically calculated spread for $\sigma$ of the order of half a nanometer: this yields an independent estimation of the level of unintentional (technology-limited) disorder, which is quite close to the previous one.

It is interesting to note that, among the calculated distributions of Fig. 9(b), there is one case in which disorder increases the quality factor of the cavity mode with respect to $\sigma=0$. In that case, a special realization of disorder improves local geometry optimization of the cavity mode, leading to a further increase of the $Q$ factor. This may be explained by the following: As light oscillates in the cavity, it encounters exactly the same disorder on each trip. This results in the formation of a distorted, but well defined, standing wave pattern, generally with different $\mathbf{k}$-vector components inside 

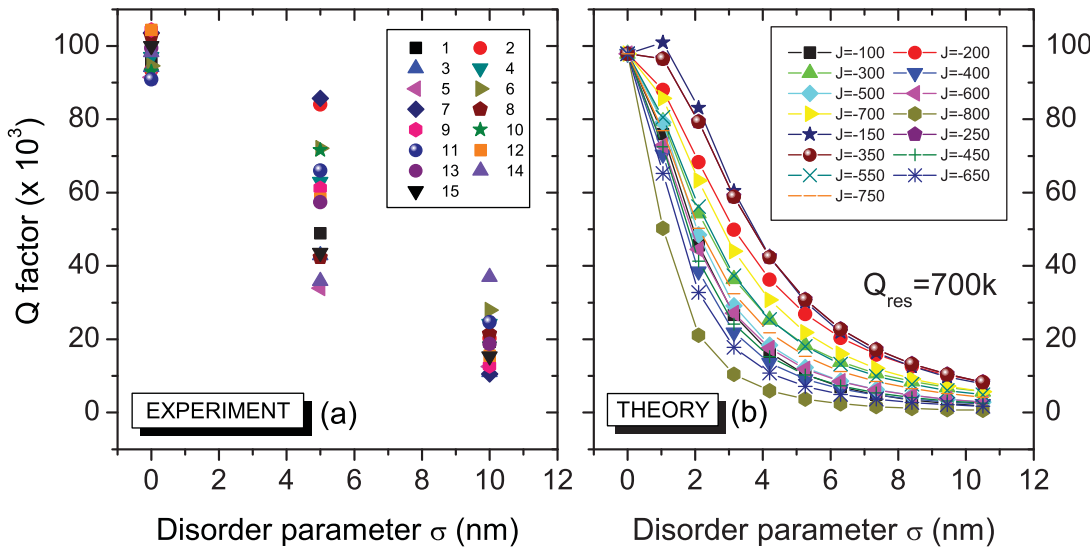

FIG. 9. (Color online) (a) Experimental behavior and (b) theoretical calculation of $Q$ factors versus the disorder parameter $\sigma$ for 15 different disorder distributions. The nearby hole shift is fixed to $\Delta x / a=0.16$. the light cone. In some instances, the k-vector distribution may be such that out-of-plane radiation is actually reduced, improving the $Q$ factor. Similar behavior is also observed experimentally, with remarkably high $Q$ factors measured for cavities with significant disorder, e.g., $Q=80 \times 10^{3}$ for $\sigma=$ $5 \mathrm{~nm}$ in Fig. 9(a).

Figure 10 shows the measured and calculated wavelengths for the same cavities of Fig. 9. Apart from a small overall shift $(\sim 5 \mathrm{~nm})$, the trend of cavity mode wavelengths versus disorder parameter is very well accounted for by theory. Most importantly, the spread of wavelengths is accurately reproduced. Again, the difference between the theoretical and experimental spreads at zero intentional disorder indicates the level of residual disorder and compares well with previous estimates. The effect of any unintentional correlation on the resonance wavelengths will also be different to that on the $Q$ factors. As the resonance wavelength is primarily given by the mode shape and volume, it will be less strongly affected by any correlation of disorder and, consequently, the agreement between theory and experiment is better. This also shows that the definition of disorder parameter $\sigma$ is consistently given in experiment and theory, a nontrivial point.

Thus, the comparison of measured and calculated wavelengths confirms the validity of the theoretical description with a one-parameter disorder model and is compatible with the previous argument about the possible presence of correlated disorder. Generally speaking, a more refined description of the effects of fabrication imperfections would require adopting a two-parameter disorder model with intrahole correlations ${ }^{23,29}$ in addition to possible inter-hole correlations resulting from stray electrons in $e$-beam lithography. Such a model is not expected to change any of the previous conclusions about the effect of disorder on cross-polarized light scattering (Sec. III) and on the general behavior of mode wavelengths, $Q$ factors, and their spread.

It is interesting to notice that the spread of wavelengths at zero intentional disorder in Fig. 10(a) is well accounted for by a disorder parameter $\sigma=1 \mathrm{~nm}$ in Fig. 10(b), while the spread of $Q$ factors at zero disorder in Fig. 9(a) corresponds to $\sigma$ much smaller than $1 \mathrm{~nm}$ in Fig. 9(b). This suggests again that wavelengths and $Q$ factors are affected in a different way by deviations from the designed disorder distribution, which we attribute to correlations due to proximity effect in lithography.

Hagino et al. have reported qualitatively similar results in their theoretical work on the effect of fluctuations in hole radii and positions in heterostructure nanocavities. ${ }^{30}$ The general trends in $Q$ factor and resonant wavelength are in good agreement with those reported here. However, there are differences in the actual values of $Q$ as a function of $\sigma$; for example, a $Q$ of $2 \times 10^{6}$ is obtained in Ref. 30 for $\sigma=$ $1 \mathrm{~nm}$, while all our values (theoretical $Q_{\text {design }}$ and residual $Q_{\text {res }}$ ) are considerably smaller. Some of these quantitative differences can be attributed to the well-known fact that the designed $Q$ factors of heterostructure cavities are generally much higher than those of L3 cavities. Nevertheless, we believe that caution is required when comparing absolute numbers for the effects of disorder in different systems and/or
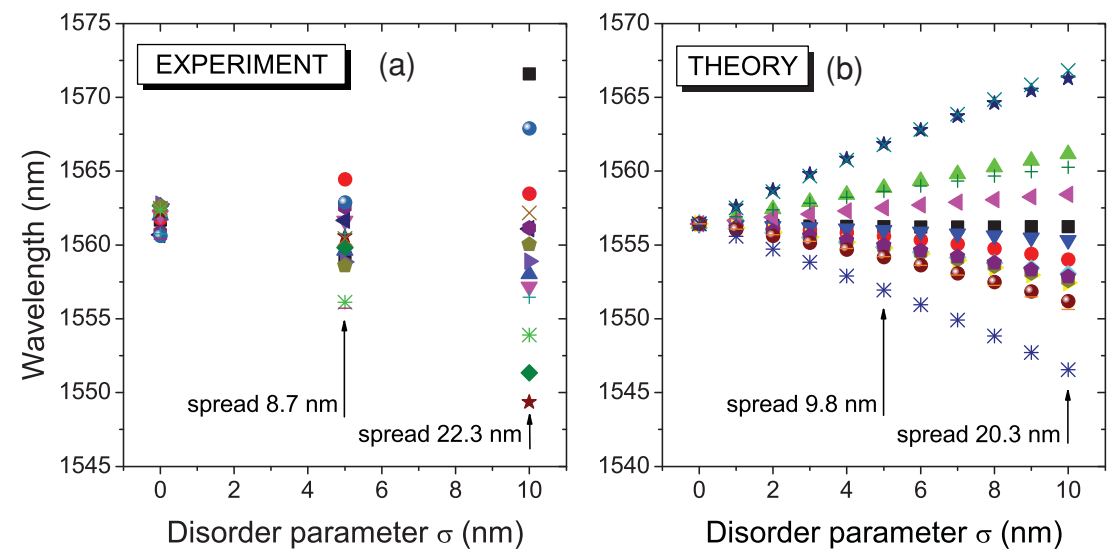

FIG. 10. (Color online) (a) Experimental behavior and (b) theoretical calculation of cavity mode wavelength versus the disorder parameter $\sigma$ for 15 different disorder distributions. The nearby hole shift is fixed to $\Delta x / a=0.16$. 
with different computational implementations (meaning realor reciprocal-space cutoffs, gridding effects, etc). The various aspects may interfere in a nontrivial way, thereby jeopardizing a precise comparison of results. The comparison with the experimental data shown in Figs. 8, 9, and 10 provides strong support for the method presented in this paper, nevertheless, since the parameter $A$ in Eq. (3) is design dependent, the application of this method to other cavity designs is nontrivial.

A comparison with waveguides $22,23,29,31-36$ is challenging for similar reasons. In a waveguide, the traveling wave always experiences a different distribution of imperfections, being subject to "truly random"disorder rather than the repeated distribution (with a period given by the roundtrip) experienced by the standing wave of the cavity. In addition, for a waveguide, some physical effects such as distribution of backscattered electrons may be different and there is also a complex interplay between backscattering and out-of-plane loss. This makes quantitative comparisons difficult at the current time.

\section{v. CONCLUSIONS}

In this paper, we have studied the effects of intentional lattice disorder on the fundamental mode of $L 3 \mathrm{PhC}$ cavities, focusing on the mode symmetry and quality factor. By using measurements of cross-polarized laser light scattering for different sample orientations, we have demonstrated that the presence of lattice disorder gives rise to a signal for every orientation, including $90^{\circ}$ and $0^{\circ}$ (i.e., cavity axis aligned with either the analyzer or the polarizer). The results are consistent with a group-theoretical analysis of cavity mode symmetry. In the absence of disorder for $90^{\circ}$ and $0^{\circ}$ orientation, there is no simultaneous coupling of cavity mode with incoming and outgoing fields, thus, the cross-polarized scattering signal is zero. In the presence of disorder, calculations of the mode profile indicate that the in-plane symmetry is broken and resonant scattering can occur for any orientation, in agreement with the experiments. Thus, cross-polarized light scattering can occur in two circumstances: when the cavity axis is tilted with respect to polarizer and analyzer axes, or when the sample contains disorder, which allows for in- and out-coupling for any orientation.

We have also demonstrated that, as might be expected, the $Q$ factors generally decrease with increasing lattice disorder, but the detailed behavior of $Q$ versus disorder parameter $\sigma$ can be quite different from one hole radii distribution to another. The spread of mode $Q$ factors and wavelengths for different values of $\sigma$ is also an interesting quantity in relation with the effects of disorder. The spread in $Q$ factor reaches a maximum for $\sigma=2.5-5 \mathrm{~nm}$ before reducing as the disorder-induced loss dominates. The resonance wavelengths behave differently, with their spread always increasing with disorder. It is particularly striking that, for certain distributions, even significant disorder causes only a small reduction in $Q$, with one cavity giving a $Q$ of $80 \times 10^{3}$ for $\sigma=5 \mathrm{~nm}$, i.e., even when the intentional disorder parameter is nearly 10 times larger than the residual one. Analysis of the results by means of theoretical calculations allows estimating the amount of residual, unintentional disorder, which ranges from $\sim 1.1$ to $0.6 \mathrm{~nm}$ for the investigated samples. We emphasize that these values, which represent the technology limits for the nanofabrication procedure, apply to a large set of cavities and, in this sense, the results are truly representative of a reproducible technology.

\section{ACKNOWLEDGMENTS}

We would like to acknowledge $\mathrm{K}$. Welna for useful discussions. This work was supported by MIUR through FIRB Contract No. RBAP06L4S5 and by ERANET project LECSIN. The fabrication was carried out in the framework of the ePIXnet Nanostructuring Platform for Photonic Integration (see http://www.nanophotonics.eu).
${ }^{1}$ D. K. Armani, T. J. Kippenberg, S. M. Spillane, and K. J. Vahala, Nature (London) 421, 925 (2003).

${ }^{2}$ B. S. Song, S. Noda, T. Asano, and Y. Akahane, Nat. Mater. 4, 207 (2005).

${ }^{3}$ T. Tanabe, M. Notomi, E. Kuramochi, A. Shinya, and H. Taniyama, Nat. Photonics 1, 49 (2007).

${ }^{4}$ S. Noda, M. Fujita, and T. Asano, Nat. Photonics 1, 449 (2007)

${ }^{5}$ M. Notomi, Rep. Prog. Phys. 73, 096501 (2010).

${ }^{6}$ Photonic Microresonator Research and Applications, Springer Series in Optical Sciences, 156, edited by I. Chremmos, O. Schwelb, and N. Uzunoglu (Springer, New York, 2010).

${ }^{7}$ T. Aoki, B. Dayan, E. Wilcut, W. P. Bowen, A. S. Parkins, T. J. Kippenberg, K. J. Vahala, and H. J. Kimble, Nature (London) 443, 671 (2006).

${ }^{8}$ K. Hennessy, A. Badolato, M. Winger, D. Gerace, M. Atatüre, S. Gulde, S. Fält, E. L. Hu, and A. Imamoğlu, Nature (London) 445, 896 (2007).
${ }^{9}$ M. Galli, D. Gerace, K. Welna, T. F: Krauss, L. O'Faolain, G. Guizzetti, and L. C. Andreani, Opt. Express 18, 26613 (2010).

${ }^{10}$ F. De Angelis, M. Patrini, G. Das, I. Maksymov, M. Galli, L. Businaro, L. C. Andreani, and E. Di Fabrizio, Nano Lett. 8, 2321 (2008).

${ }^{11}$ A. Di Falco, L. O'Faolain, and T. F. Krauss, Appl. Phys. Lett. 94, 063503 (2009).

${ }^{12}$ Y. Akahane, T. Asano, B. S. Song, and S. Noda, Nature (London) 425, 944 (2003).

${ }^{13}$ L. C. Andreani, D. Gerace, and M. Agio, Photon. Nanostr. Fund. Appl. 2, 103 (2004).

${ }^{14}$ C. Sauvan, P. Lalanne, and J. P. Hugonin, Phys. Rev. B 71, 165118 (2005).

${ }^{15}$ M. McCutcheon, G. W. Rieger, I. W. Cheung, J. F. Young, D. Dalacu, S. Frédéric, P. J. Poole, G. C. Aers, and R. Williams, Appl. Phys. Lett. 87, 221110 (2005).

${ }^{16}$ M. Galli, S. L. Portalupi, M. Belotti, L. C. Andreani, L. O’Faolain, and T. F. Krauss, Appl. Phys. Lett. 94, 071101 (2009). 
${ }^{17}$ P. Deotare, M. McCutcheon, I. Frank, M. Khan, and M. Lončar, Appl. Phys. Lett. 94, 121106 (2009).

${ }^{18}$ U. Fano, Phys. Rev. 124, 1866 (1961).

${ }^{19}$ L. O'Faolain, X. Yuan, D. McIntyre, S. Thoms, R. M. De La Rue, and T. F. Krauss, Electron. Lett. 42, 1454 (2006).

${ }^{20}$ M. Spurny, L. O'Faolain, D. A. P. Bulla, B. Luther-Davies, and T. F. Krauss,Opt. Express 19, 1991 (2011).

${ }^{21}$ L. C. Andreani and D. Gerace, Phys. Rev. B 73, 235114 (2006).

${ }^{22}$ D. Gerace and L. C. Andreani, Photon. Nanostr. 3, 120 (2005).

${ }^{23}$ L. C. Andreani and D. Gerace, Phys. Status Solidi B 244, 3528 (2007).

${ }^{24}$ K. Sakoda, Optical Properties of Photonic Crystals (Springer, New York, 2001).

${ }^{25} \mathrm{~T}$. Inui, Y. Tanabe, and Y. Onodera, Group Theory and Its Applications in Physics (Springer, New York, 1996).

${ }^{26}$ In Ref. 16, we considered only the case of no intentional disorder and denoted $Q_{\text {design }}(\sigma \rightarrow 0) \equiv Q_{0}$.

${ }^{27}$ See Fig. 8(b) of Ref. 22. The formula in the text is valid in the perturbative limit, which holds for the small values of residual disorder. The nonperturbative behavior mentioned in Sec. IIC occurs for values of $\sigma$ larger than a few nanometers.
${ }^{28}$ R. Wüest, P. Strasser, M. Jungo, F. Robin, D. Erni, and H. Jäckel, Microelectron. Eng. 67-68, 182 (2003).

${ }^{29}$ S. Hughes, L. Ramunno, J. F. Young, and J. E. Sipe, Phys. Rev. Lett. 94, 033903 (2005).

${ }^{30}$ H. Hagino, Y. Takahashi, Y. Tanaka, T. Asano, and S. Noda, Phys. Rev. B 79, 085112 (2009).

${ }^{31}$ J. Topolancik, B. Ilic, and F. Vollmer, Phys. Rev. Lett. 99, 253901 (2007).

${ }^{32}$ T. P. White, L. O'Faolain, J. Li, L. C. Andreani, and T. F. Krauss, Opt. Express 16, 17076 (2008).

${ }^{33}$ M. Patterson, S. Hughes, S. Schulz, D. M. Beggs, T. P. White, L. O'Faolain, and T. F. Krauss, Phys. Rev. B 80, 195305 (2009).

${ }^{34}$ M. Patterson, S. Hughes, S. Combrié, N.-V.-Quynh Tran, A. De Rossi, R. Gabet, and Y. Jaouën, Phys. Rev. Lett. 102, 253903 (2009).

${ }^{35}$ S. Mazoyer, J. P. Hugonin, and P. Lalanne, Phys. Rev. Lett. 103, 063903 (2009).

${ }^{36}$ L. O'Faolain, S. A. Schulz, D. M. Beggs, T. P. White, M. Spasenovic, L. Kuipers, F. Morichetti, A. Melloni, S. Mazoyer, J. P. Hugonin, P. Lalanne, and T. F. Krauss, Opt. Express 18, 27627 (2010). 